\title{
Rats' object-in-place encoding and the effect of fornix transection
}

\author{
E. L. SIMPSON and E. A. GAFFAN \\ University of Reading, Reading, England \\ and \\ M. J. EACOTT \\ University of Durham, Durham, England
}

\begin{abstract}
We studied the perception of simple computer-generated scenes by normal and fornix-transected Dark Agouti rats. In Experiment 1, the rats were rewarded for approaching trial-unique variable scenes differing from a constant scene that was the same across trials (constant-negative paradigm). The groups performed equivalently when scenes differed only in their objects or only in the occupied positions; however, when two scenes shared an object-place combination, the normal rats were more likely to see them as similar than were the fornix-transected rats. In Experiment 2, the rats learned to discriminate pairs of scenes. Again, there was no lesion effect when scenes differed by a single cue, object or position, but when the two scenes comprised the same objects interchanged in position, fornixtransected rats learned relatively easily. Fornix transection reduces rats' sensitivity to object-place combinations within scenes, consistent with D. Gaffan's account of scene memory as an animal analogue of episodic memory deficits in amnesia.
\end{abstract}

Lesions of the hippocampus or of its major input or output systems affect many aspects of animals' spatial learning (Jarrard, 1995; Sutherland \& Rodriguez, 1989). One of these is knowledge about the location of objects in the environment. Rats with hippocampectomy or fornix transection are less likely than normals to renew exploration of objects that have been moved or spatially rearranged (Ennaceur, Neave, \& Aggleton, 1997; Save, Poucet, Foreman, \& Buhot, 1992) and are impaired in tasks that reward them for remembering object-place associations (Long \& Kesner, 1995), although there is also evidence that they are insensitive to place cues in this paradigm (DeCoteau \& Kesner, 1998). Monkeys with ablations of the hippocampus or of the parahippocampal cortex perform poorly when required to recognize which of a pair of familiar objects has moved to a new place (Angeli, Murray, \& Mishkin, 1993; Malkova \& Mishkin, 1997; Parkinson, Murray, \& Mishkin, 1988), and fornix transection disrupts their yes-no recognition of object-place combinations (D. Gaffan \& Saunders, 1985). Similarly, people with lesions of the hippocampus or of the parahippocampal cortex, particularly in the right hemisphere, show deficits when they are asked to remember the spatial layout of items within a visually presented scene (Nadel, Allen, Bohbot, Kalina, \& Stepankova, 1997; Pig-

This research was supported by the U.K. Medical Research Council. We thank Steve Nagle for valuable assistance. Correspondence concerning this article should be addressed to E. A. Gaffan, Department of Psychology, University of Reading, Reading RG6 6AL, England (email: e.a.gaffan@reading.ac.uk). ott \& Milner, 1993; Smith \& Milner, 1989). Scanning studies of normal people performing analogous tasks, which test memory for the locations at which objects have previously appeared, implicate the parahippocampal cortex (Milner, Johnsrude, \& Crane, 1997; Owen, Milner, Petrides, \& Evans, 1996). Although there are many differences between the details of the tasks and between their sensory and motor requirements, it seems that, in both animals and people, memory for where objects are located in space relies on an intact hippocampal system.

It has been suggested by D. Gaffan that the ability to remember the spatial layout of objects within a scene is central to human episodic memory and that tasks that tax that ability constitute an animal model of episodic memory impairment in amnesia (D. Gaffan, 1992b, 1994). He has studied monkeys with lesions of the fornix, mamillary nuclei, or anterior thalamus, all of which are hippocampal projection targets and are implicated in amnesia (Aggleton \& Sahgal, 1993; D. Gaffan \& E. A. Gaffan, 1991; Kapur et al., 1994). The tasks employ computergenerated abstract displays in which "objects" (colored shapes) are displayed against large complex backgrounds made up of a number of colored areas. Monkeys with the aforementioned lesions are deficient in learning to choose between pairs of objects when each pair is displayed against its own unique background or "scene." The impairment is clearest when each object of a pair occupies a fixed position within its scene - this is described as objectin-place memory (D. Gaffan, 1994; Parker \& D. Gaffan, $1997 \mathrm{a}, 1997 \mathrm{~b}$ ). That is striking, because the object-inplace variant of the task is easier than any other for intact monkeys. By contrast, when monkeys must discriminate 
between comparable pairs of objects presented against backgrounds that vary from trial to trial, fornix transection produces no impairment (D. Gaffan, 1994). Hornak, Oxbury, Oxbury, Iversen, and D. Gaffan (1997) have recently shown that patients who have undergone unilateral hippocampal surgery for treatment of epilepsy are impaired at remembering analogous object-in-place displays in the visual field that projects to the hemisphere with the hippocampal lesion.

So there is evidence that normal monkeys are adept at remembering where objects have appeared within a complex scene and that this kind of memory is particularly sensitive to lesions that cause amnesia in people. In D. Gaffan's scene memory tasks, subjects are not required explicitly to remember the location at which objects have occurred - they merely have to learn which scene, or object within a scene, they must touch to obtain a rewardbut there is a plausible analogy between these findings and the specific deficits of object-location memory in rats and monkeys described previously. Gaffan notes the connection with other tasks, also sensitive to fornix transection, in which monkeys must choose between objects, conditional on the particular spatial arrangement of the objects (D. Gaffan, 1992a; D. Gaffan \& Harrison, 1989a, 1989b).

In our experiments, we asked whether normal rats, like normal monkeys, spontaneously encode the objectplace combinations within visual scenes and whether this encoding is altered by transection of the fornix. Note that we tested encoding rather than memory as such. Encoding is fundamental to memory, although, of course, memory might be impaired even when encoding is not (Smith \& Milner, 1989).

The rats were tested in an apparatus in which they discriminate between wide-angle computer-generated visual scenes, analogous to those used with monkeys, whose contents can be varied and controlled (E. A. Gaffan \& Eacott, 1995). We investigated their perception of similarity between pairs of scenes that could differ in the objects that they contained and/or in the spatial location of the objects. Thus, we manipulated the object and spatial features of the scenes in a more explicit way than was done in the monkey scene memory studies. Another difference is that, in D. Gaffan's apparatus for monkeys, the scenes were shown on a fixed screen, which the monkey viewed from a fixed direction. In our apparatus, which is a Y-maze, any scene can be displayed in any of the three arms, varying from trial to trial. Thus, the spatial relationships that we manipulated were defined within the scenes themselves but not stably related to fixed places within the room.

E. A. Gaffan and Eacott (1997), using the same apparatus and a simple discrimination paradigm, showed that, with very simple scenes containing only one object, there was no difference between fornix-transected rats and controls in encoding the position of a single object. In the present study, the scenes contained two objects, so we were able to ask more complex questions about scene encoding. When designing these tasks, it is important to distinguish between scenes that could be discriminated solely on the basis of the elements that they containobjects or positions-and scenes in which the elements do not differ but the object-position combinations do. This was achieved in slightly different ways in the two experiments.

In Experiment 1, we used the constant-negative discrimination paradigm (Cassaday \& E. A. Gaffan, 1996; E. A. Gaffan \& Woolmore, 1996). Within each problem, one scene (the constant) is the same on every trial, whereas the other (the variable) is different on every trial, and the rat is rewarded for approaching the variable scene. We manipulated the features of the variable scenes across trials; for example, a variable might contain the same objects as the constant but in a different spatial arrangement (for full details, see the Method section). If a particular kind of manipulation makes a variable scene appear to be similar to the constant, the rats should discriminate poorly on trials in which that kind of variable is presented.

Thus, in Experiment 1, variable scenes might differ from the constant in any of a number of ways, and we tested whether the rats spontaneously found some sorts of difference more salient than others. In Experiment 2, the task was simple discrimination learning between a fixed pair of scenes that differed in only one respect-for example, only in the objects that they contained or only in the object-position combinations. We tested whether the fornix-transected and control rats differed in the ease of using particular kinds of cue.

\section{EXPERIMENT 1}

\section{Method}

Subjects. Fourteen male rats of the pigmented Dark Agouti strain (Aggleton, 1996) took part in the experiment. They were 4 months of age at surgery and 5 months at the start of training. In 5 rats (Group FX), the fornix was transected, whereas 9 rats (Group SH) were shamoperated controls. The rats were housed in pairs with free access to water and were maintained at or above $85 \%$ of their ad-lib weight by feeding after experimental sessions. The light cycle was $12: 12-\mathrm{h}$ light:dark, and behavioral testing took place during the light phase.

Apparatus. Two computer-controlled Y-mazes were used (see E. A. Gaffan \& Eacott, 1995). The two mazes were similar in overall dimensions and equipment, but manufacturers of components sometimes differed between the mazes. Figure 1 represents the floor plan of one maze; the other was very similar. Roughly half of the rats in each group were trained in each maze.

Each of the three arms was equipped with two monochrome VGA monitors placed side by side at a shallow angle, $43 \mathrm{~cm}$ from the maze center, providing a total image area $47 \mathrm{~cm}$ wide $\times 18.5 \mathrm{~cm}$ high, which subtended about $94^{\circ}$ horizontally at the maze center. In each arm, in the space between the two screens, was a foodtray, into which 45-mg diet pellets were dropped from a dispenser (Campden Instruments, Loughborough, England or CeNeS, Ely, England) located above the maze. The brand of food pellets was either Bioserv (Campden Instruments) or Noyes Type P (Sandown Scientific, Esher, England). The magazine could be internally illuminated. 


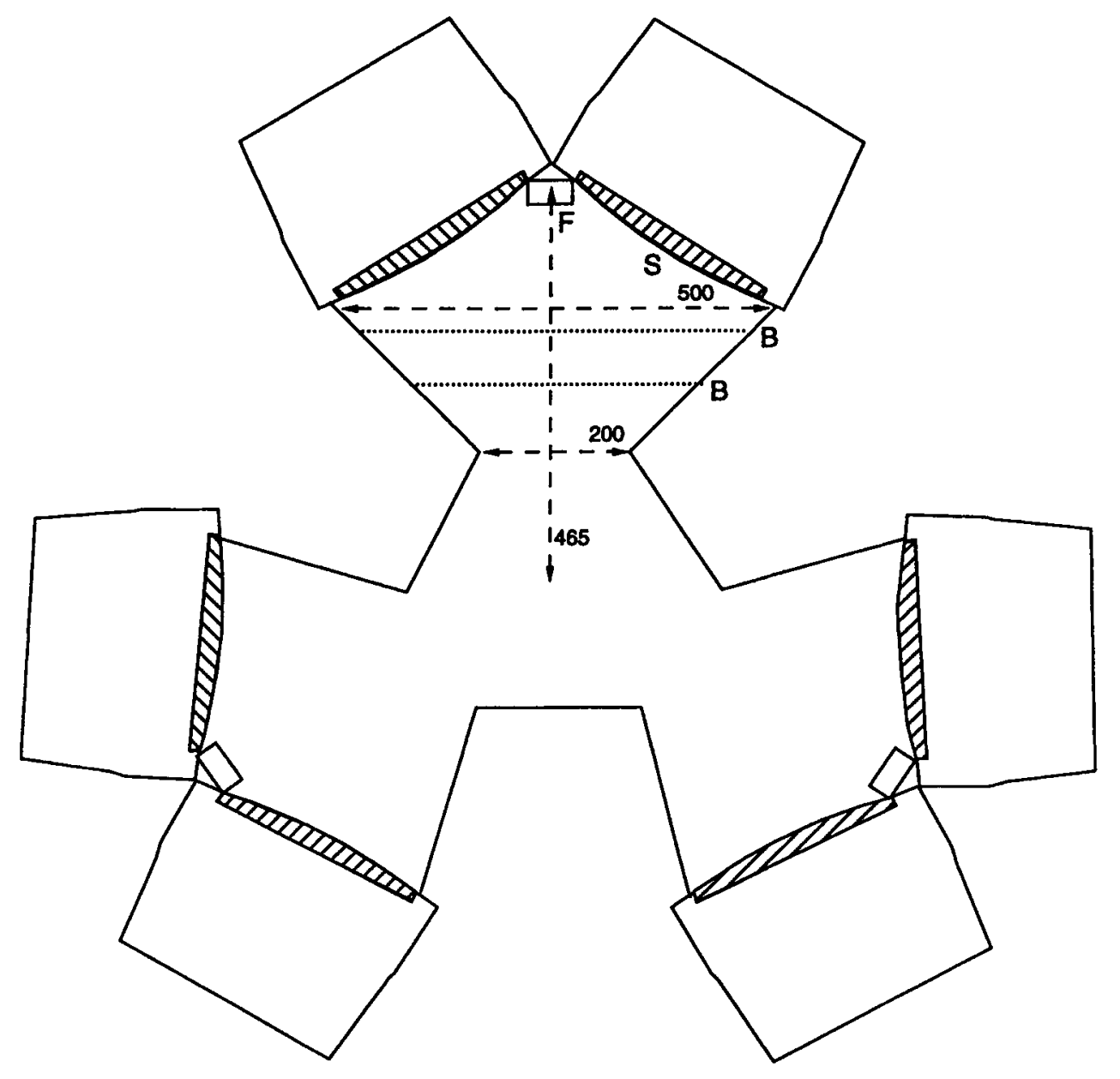

Figure 1. View of the floor plan of the maze. All three arms are identical; only one is shown in detail. Dashed lines are dimensions, in millimeters; dotted lines show the positions of the photodetector beams. F, feeder; S, monitor screen; B, photodetector beam.

Each arm was crossed by two infrared photodetector beams, 23 and $30 \mathrm{~cm}$ from the maze center, to monitor the rat's presence in the arms. The transparent flap in front of the foodtray was fitted with a microswitch to detect tray entries. The maze was painted matte black and roofed with Plexiglas. The room was dimly lit, and overhead video cameras allowed the experimenter to monitor events from an adjacent room. All inputs and outputs, including stimulus presentation, were handled by a 486DX IBM-compatible computer (one for each maze) fitted with three Dual VGA Plus or Warp 2 video cards (Colorgraphics Communications, Atlanta), each capable of independently controlling two VGA monitors. Control programs were written in Turbo Pascal 6.0.

Surgery and histology. Each rat was anesthetized by intraperitoneal (i.p.) injection of pentobarbital sodium (Sagatal) at $60 \mathrm{mg} / \mathrm{kg}$, with further subcutaneous doses of Sagatal as needed to maintain anesthesia during surgery. The rat was placed in a stereotaxic headholder (David Kopf, Tujunga, CA). The scalp was retracted, a small craniotomy was made, and the dura was slit to expose the cortex above the target region. Five of the rats received radiofrequency lesions of the fornix bilaterally. A Radionics TCZ electrode $(0.3-\mathrm{mm}$ tip length, $0.25 \mathrm{~mm}$ in diameter; Radionics, Burlington, VT) was lowered into two sites in each hemisphere. The lesion coordinates relative to ear-bar zero, with the incisor bar set at +5.0 , were AP +5.3 , LAT $\pm 0.7,3.7 \mathrm{~mm}$ below the top of the dura; and AP -0.4 , LAT $\pm 1.8,3.8 \mathrm{~mm}$ below the top of the dura. The temperature of the electrode tip was raised to $75^{\circ}$ for $60 \mathrm{sec}$, using an RFG4-A lesion maker (Radionics). The remaining 9 rats underwent sham surgery; they were anesthetized, the incision was made, and the electrode lowered into the brain, but radiofrequency lesions were not made. The wounds were closed and sealed with wound clips, and powder containing $20 \%$ chloramine was applied.

After completion of behavioral testing, the rats were given an overdose of Sagatal $(0.35 \mathrm{ml}, 60 \mathrm{mg} / \mathrm{kg}$, i.p. $)$ and transcardially perfused with saline, followed by $10 \%$ formol-saline. The brains were removed and post-fixed in formol-saline. Coronal sections were cut at $60 \mu \mathrm{m}$ and stained with cresyl violet.

Stimuli.The stimuli were computer-generated scenes, in shades of gray ranging in luminance from black up to $5.0 \mathrm{~cd} / \mathrm{m}^{2}$. Each scene extended across the two adjacent monitors within one arm of the maze and comprised objects (abstract shapes) distributed across 
a background of contrasting brightness. The scenes varied slightly across stages of training. Examples of scenes used in Experiment 1 are shown in Figures 2 and 3.

In the figures, each panel represents the two monitor screens side by side. Each scene contains two objects, either both on one screen or one per screen, against a uniform background. In these examples, the background is light gray and the objects in various darker grays, but it was also possible for the background to be dark and the objects lighter. The objects were sampled from a pool of 240 shapes, including geometric forms (ellipses, rectangles, polygons, disk segments) and large alphanumeric characters. About 1/3 of the objects were single shapes; the remainder were 2 similar shapes superimposed (e.g., a large and a smaller rectangle at different orientations). The luminances varied as follows. If the background was dark $\left(\sim 0.01 \mathrm{~cd} / \mathrm{m}^{2}\right)$, the grays used to display the objects varied between 0.27 and $5.0 \mathrm{~cd} / \mathrm{m}^{2}$. If the background was light $\left(5 \mathrm{~cd} / \mathrm{m}^{2}\right)$, the objects' grays ranged from 0.02 to $0.61 \mathrm{~cd} / \mathrm{m}^{2}$. The sizes of the objects varied, the smallest being about $40 \mathrm{~mm}$ in maximum extent and the largest about $75 \mathrm{~mm}$. The objects could be centered at any one of 32 positions, 16 on each screen. The 16 positions formed a $4 \times 4$ grid, $35 \mathrm{~mm}$ apart and starting $40 \mathrm{~mm}$ from the inner edge of the screens, closest to the feeder. The outermost parts of the screens were not used, because pilot work had shown that rats might fail to notice isolated objects at extreme lateral positions.

In each problem that the rats learned, comprising a constant scene and many variable scenes, all the scenes had the same background brightness, light or dark. The constant scene was constructed by choosing two different objects and placing them at 2 of the 32 positions. Eighty variable scenes, all different from each other, were constructed for each session. There were five possible types of variable scene - called N, O2, P2, OP1, and O1P1-defined by the nature of their similarity to the constant scene (see below). Figures 2 and 3 show examples of the five types.

Type $N$ (no similarity). The variable scene comprised two objects that were different from both objects in the constant, at two positions different from those used in the constant. The positions used in the variable were half the time on two different screens and half the time both on one screen (see Figures 2 and 3).

Type $\mathrm{O} 2$ (two objects same). The variable scene comprised the same two objects as did the constant, but placed at two different positions, chosen as for type N (see Figure 2).

Type P2 (two positions same). The variable scene used the same two positions as did the constant, but both objects were different (see Figure 2).

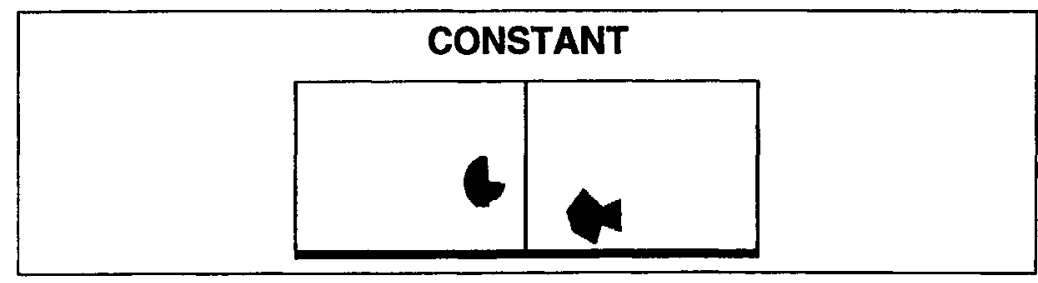

VARIABLES:

Type $\mathbf{N}$ (no similarity)

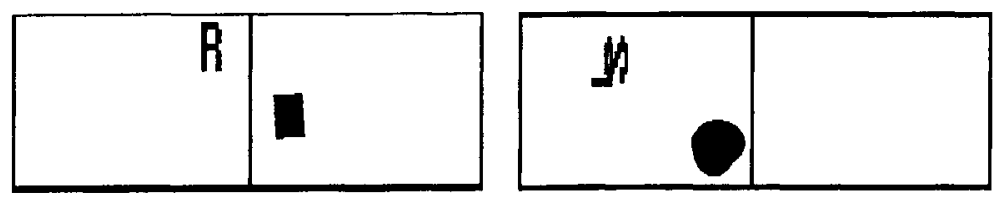

Type P2 (both positions same)
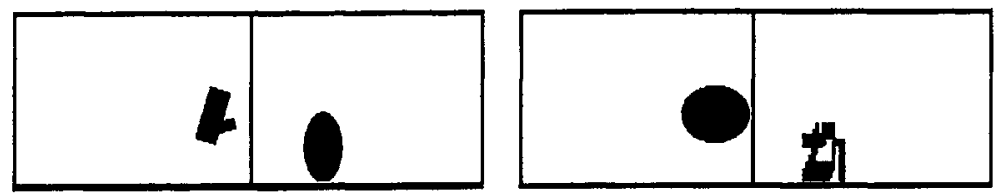

Type 02 (both objects same)
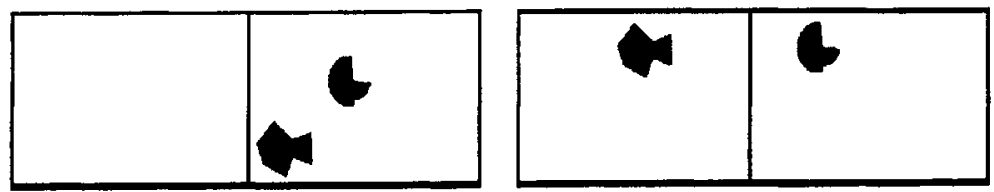

Figure 2. Examples of scenes used in Experiment 1. A constant scene and two examples of three types of variable scene that might be associated with that constanttype $N$, type $P 2$, and type $O 2$. A different variable scene was shown on every trial. See the text for details. 


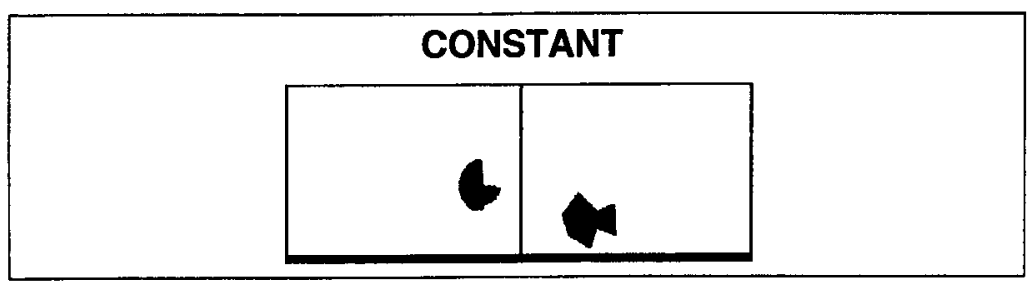

VARIABLES:

Type N (no similarity)

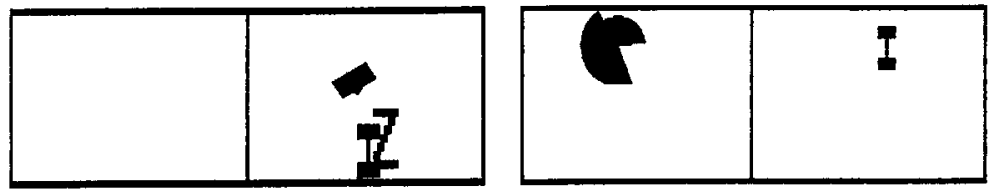

Type OP1 (1 object-position compound same)

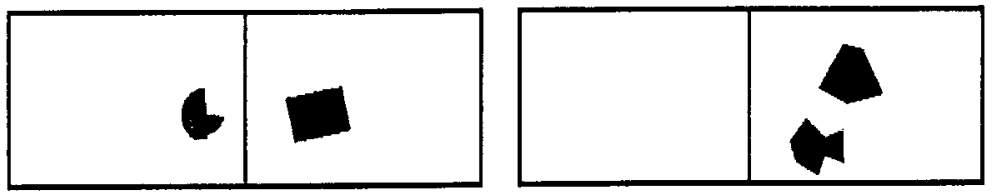

Type 01P1 (1 object \& 1 position same, not compounded)

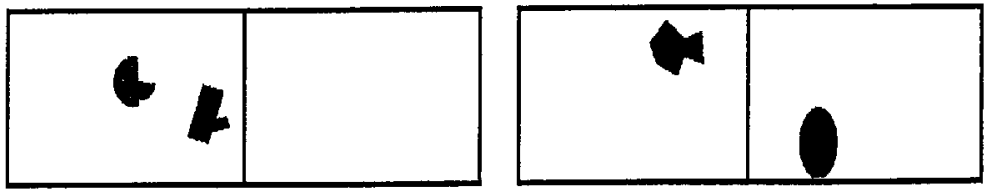

Figure 3. The same constant scene as that in Figure 2, with two examples of three types of variable scene-types $\mathrm{N}, \mathrm{OP} 1$, and $\mathrm{O1P1}$.

Type OPI (one object-position compound same). One of the objects in the variable scene was the same object, in the same position, as one of those in the constant-that is, it exactly matched between the two scenes. The other was a different object in a different position. The matching object-position compound could be either one (see Figure 3).

Type OIPI (one object same, one position same, not compounded). One of the objects in the variable matched one in the constant but was at a different position. The second object was different from either in the constant but was placed at the same position as the other (nonmatching) object of the constant. Thus, one object and one position in the variable also occurred in the constant, but not combined together (see Figure 3). This was designed as a control for type OP1, having the same amount of similarity in individual elements (one object, one position) but being less similar in terms of object-position combinations.

In all cases, sampling of new objects and positions was random (controlled by a random seed that was specific to the problem and the session) but constrained. New objects were not used more than once per session and differed in as many ways as possible (form, size, brightness) from the corresponding object in the constant. New positions were chosen, so that variable objects were half the time within the same screen and half the time on different screens, and, if on the same screen, did not overlap.

Within each session, the variables included a specified range of types - for example, all N, or a mixture of N, OP1, and O1P1 (see the Main Experiment sections below). When several types occurred within the same session, we could compare discrimination accuracy across the types and, thus, judge what type of variable was perceived as being most similar to the constant.

Constant-negative pretraining. All behavioral training took place after fornix or sham surgery. The rats were first trained to collect food pellets from any illuminated foodtray and to approach a particular foodtray when a standard signal (a white horizontal stripe extending across both screens) was displayed in that arm (see E. A. Gaffan \& Eacott, 1995, or E. A. Gaffan \& Woolmore, 1996, for details of initial training). They then learned a series of problems of the constant-negative discrimination task, using progressively more demanding stimuli. Training on a given problem could continue across more than one session, depending on how long the rat took to reach criterion.

The procedure is schematized in Figure 4. A session comprised 2 preliminary errorless trials, followed by up to 80 constant-versusvariable choice trials. In each trial, one arm was designated the start 

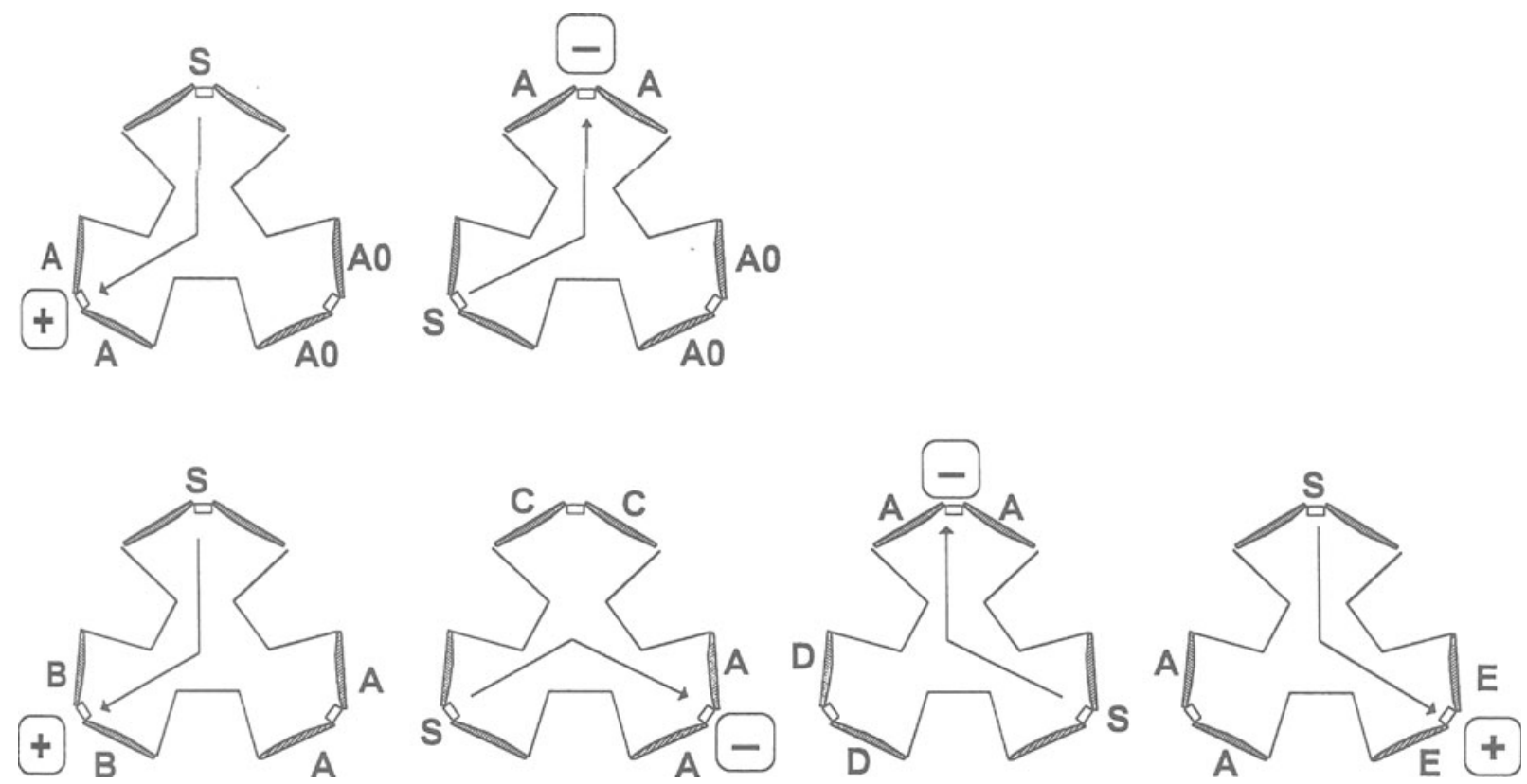

Figure 4. Examples of successive trials of the constant-negative procedure of Experiment 1 . In each trial, $S$ is the start arm, and the constant scene $A$ is presented in one of the remaining arms. The first row represents two errorless trials that occur at the start of every session. The choice is between the constant scene (A) and blank gray screens (A0). Approaching A0 has no consequences. Approaching the constant $A$ is followed by reward $(+)$ on the first errorless trial, but terminates the trial without consequences $(-)$ on the second trial. Except on the very first trial, the start arm is the arm that the rat chose on the preceding trial. In all trials after the two errorless ones (second row), the choice is between $A$ and a trial-unique variable scene $B, C, D \ldots$, with choice of the variable being rewarded.

arm (marked $S$ in Figure 4), and, when the rat entered it, two scenes were presented in the other two arms, with their left-right position, relative to the start arm, randomized. On the first trial of a session, one of the three arms was randomly designated as the start arm and indicated to the rat by displaying the horizontal stripe signal; in all later trials, the start arm was the arm that the rat had chosen on the preceding trial (see Figure 4).

On the errorless trials (top line of Figure 4), the rat was presented with the constant scene (A), versus blank screens of similar average brightness (A0); approaching the blank screens had no consequence. On errorless Trial 1, entering the arm with the constant scene was rewarded with one food pellet-thus, the first time the constant appeared in a session, when it was relatively novel, it was rewarded. Both stimuli disappeared; then, errorless Trial 2 was presented. In this trial, approaching the constant scene simply terminated the trial without reward, both scenes being turned off. Thus, when the constant was no longer novel, it was not rewarded.

In all the subsequent trials, the rat had to choose between the constant $A$ and a variable scene that was different on every trial (represented by B, C, D... in the second row of Figure 4). Choice of the variable was rewarded with two food pellets, after which the next trial commenced without delay. If the rat chose the constant, both stimuli immediately turned off without reward, and a 2-sec delay began. After the delay had elapsed and the rat was next detected in the new start arm (i.e., the arm it had previously chosen), the next trial commenced, with a new variable scene. There was no opportunity to correct errors.

The session ended after a maximum of 80 constant-variable choice trials. If the rat attained a criterion of learning (see below), the session ended, and, in the next session, a new problem started, with a new constant scene. If it had not reached criterion after 80 choice trials, the problem continued in the following session.
In Stage 1 of pretraining, the rats learned eight constant-negative problems. The stimuli were randomly sampled from 120 scenes that were more diverse than those shown in Figures 2 and 3 . They contained varying numbers of shapes distributed across heterogeneous backgrounds. Dark Agouti rats learn constant-negative problems very easily with scenes of this type (Cassaday \& E. A. Gaffan, 1996; E. A. Gaffan \& Woolmore, 1996). For the first four problems, the criterion of learning was $80 \%$ correct across five consecutive 10 trial blocks; in the next four problems, this was raised to $85 \%$. The eight problems employed eight different constant scenes that were given in different orders to different rats within the FX and SH groups.

In Stage 2 of pretraining, the rats learned 20 problems using scenes that were more like those shown in Figures 2 and 3. They were made from the 240 shapes to be used in the main experiment, distributed across plain backgrounds. In half the problems, all the scenes had dark backgrounds and light foreground shapes; in half, vice versa. (The luminance of the brightest gray was $6.8 \mathrm{~cd} / \mathrm{m}^{2}$, higher than the final value to be used in the main experiment.) Across the 20 problems, the scenes were made progressively harder to discriminate, by making the numbers of shapes in the constant and variable scenes more and more similar, because in the main experiment all the scenes would contain 2 shapes. The learning criterion was $80 \%$ correct over 50 trials.

Main experiment, Phase 1: Postcriterion testing. The rats learned two different problems, Nos. 1 and 2, using scenes of the type shown in Figures 2 and 3 . We compared performance with the five types of variable scene defined in the Stimuli section. The highest luminance was reduced from 6.8 to $5.0 \mathrm{~cd} / \mathrm{m}^{2}$, because the rats had performed less well with light than with dark backgrounds in the preceding stage (see the Results section).

The background was light in one problem and dark in the other, in counterbalanced order. Different pairs of constant scenes were 
used for different rats, each pair being allocated to 1 or $2 \mathrm{FX}$ and 1 or $2 \mathrm{SH}$ rats. Within each pair of constant scenes, one scene had an object on each screen (like the example in Figures 2 and 3), whereas the other had two objects on the same screen.

In each problem, the rat was first trained to criterion in 80-trial sessions in which all the variables were of type $\mathrm{N}$ - that is, they were not similar to the constant in either the objects or their locations and, thus, were easy to discriminate from it. Once a criterion of $80 \%$ correct was attained in two successive sessions, a series of postcriterion test sessions began in which the 80 trials were divided between type $\mathrm{N}$ and two other types, as detailed below. Testing with a particular combination of variable types continued until three sessions had accumulated in which the rat scored at least $80 \%$ correct on the trials with variable type N. Performance with the other types of variables was recorded only from these three sessions; thus, type $\mathrm{N}$ provided a stable, high baseline against which performance with other types of variable could be compared. Within each type of variable, other features were randomized (e.g., when positions were different from those in the constant scene, the objects were sometimes on opposite screens and sometimes within the same screen).

In the first block of tests, each session comprised 40 trials with variable type $\mathrm{N}, 20$ with type $\mathrm{OP} 1$, and 20 with type $\mathrm{O} 1 \mathrm{P} 1$, in quasirandom order. If normal rats find scenes that share an object-position compound to be more similar than scenes that do not, they should perform worse (discriminate more poorly between variable and constant) on OPI trials than on O1P1 trials. If lesioned rats are deficient in encoding object-position compounds but discriminate the elements (objects and positions) as well as normals, they should perform similarly on the two types of trial.

In the second block of tests, sessions comprised $40 \mathrm{~N}, 20 \mathrm{O} 2$, and $20 \mathrm{P} 2$ trials. The purpose was to compare the groups on encoding of the elements - object or position. For example, if the rats in the lesioned group were relatively insensitive to position differences, they should perform worse than controls on $\mathrm{O} 2$ trials, in which both objects in the variable were the same as those in the constant and only the positions differed between scenes.

Main experiment, Phase 2: Precriterion testing. In the previous phase, performance had been tested only after the rats had reached criterion and, therefore, were highly familiar with the constant scene. In this phase, performance on the two most theoretically interesting types of variable, OP1 and O1P1, was compared against that on type $\mathrm{N}$ at an earlier stage of learning.

The rats again learned two problems, Nos. 3 and 4 . New constant scenes were constructed and given in a counterbalanced manner to rats from the $\mathrm{FX}$ and $\mathrm{SH}$ groups, with dark and light backgrounds also counterbalanced. During the first session of a problem, variable type $\mathrm{N}$ was given on all 80 trials, so as not to introduce the more difficult OP 1 and O1P1 at the very start of training. From Session 2 onwards, each session comprised 40 trials with type $\mathrm{N}$ variables, 20 with type OP1, and 20 with type O1P1. Training continued until the first session in which the rat scored $80 \%$ or more correct on the $\mathrm{N}$ trials.

\section{Results}

Histology. All the rats in the FX group sustained bilateral transection of the fornix at one level at least (see Figure 5). There was minor encroachment into the dorsal hippocampus. In 1 animal, there was some damage to the frontal cortex at the anterior extent of the lesion, but this was unilateral. All 5 rats were retained in the analysis.

Constant-negative pretraining. During the first four problems, with highly discriminable scenes and an $80 \%$ criterion, the mean numbers of trials taken to reach criterion by the FX and SH groups, respectively, were 110.5 and $110(t<1)$. In the next four problems, with similar stimuli and an $85 \%$ criterion, the groups took 119.5 and 156.9 trials, respectively, on the average $[t(12)=1.07$, n.s.]. Thus, fornix transection had no adverse effect on learning the basic constant-negative task.

In the second stage of pretraining, in which the scenes comprised several objects on uniform backgrounds, 20 problems were learned, half with light and half with dark backgrounds. The FX rats took, on the average, 153 trials to reach criterion in problems with dark backgrounds and 246 trials in problems with light backgrounds. The SH rats' mean scores were 158 trials with dark backgrounds and 198 trials with light. A $2 \times 2$ mixed design analysis of variance (ANOVA; groups $\times$ background brightness) showed that problems with light backgrounds were learned significantly more slowly than dark background problems $[F(1,12)=27.4, p<.001]$. The groups did not differ on the average $[F(1,12)=1.23]$, but the group $\times$ background interaction approached significance $[F(1,12)$ $=4.19, p=.06]$. The lesion groups learned dark background problems at equal rates, but the FX group was slightly slower on the more difficult light background

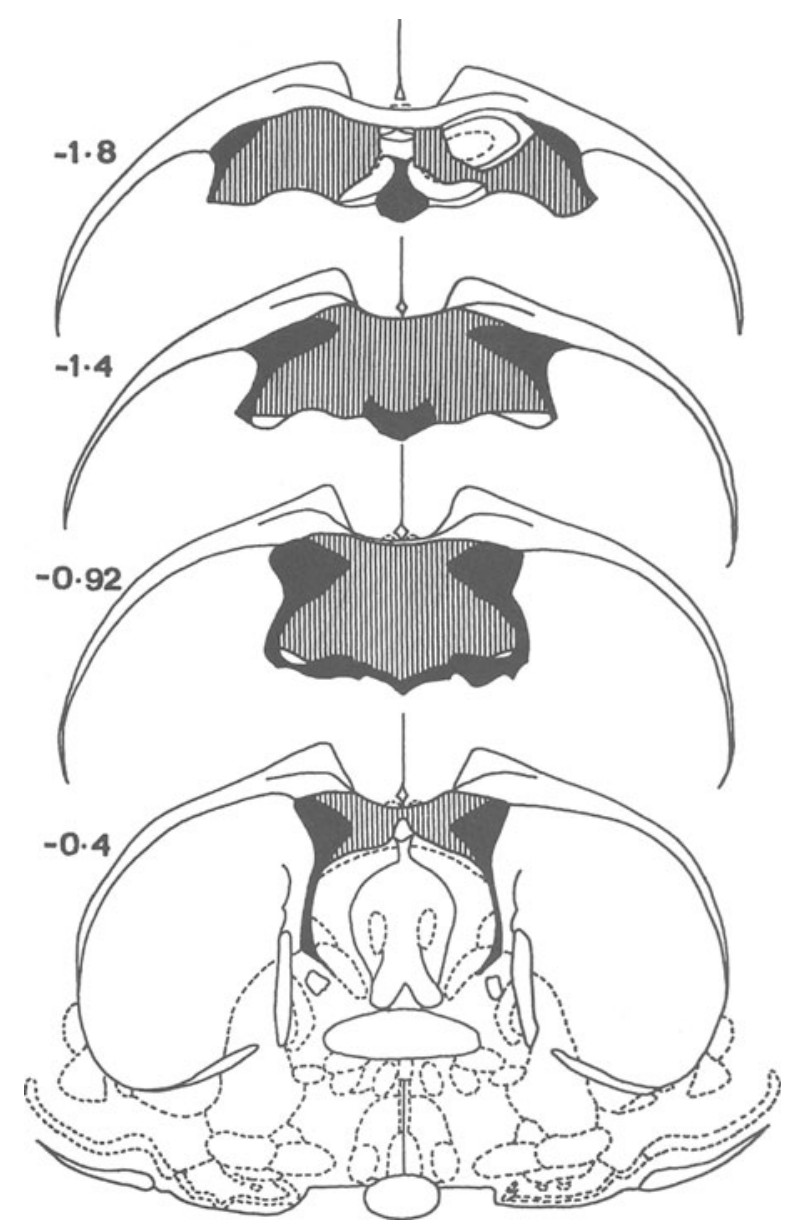

Figure 5. A series of coronal sections illustrating a typical fornix transection. Black, ventricle; hatched areas, lesion. 


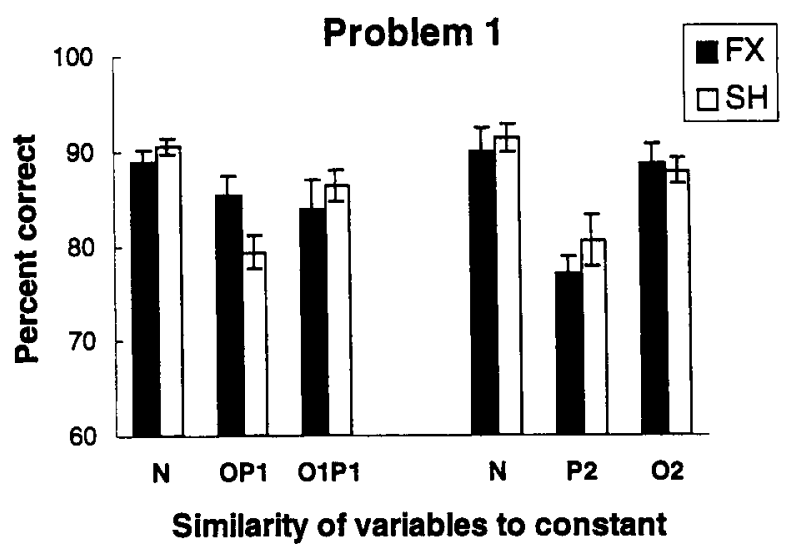

Figure 6. Experiment 1, Problem 1. Percent correct choices by the two groups (dark bars, FX; light bars, SH) on trials with different types of variable scene, in Block 1 of testing (left) and in Block 2 (right). Data are taken from three postcriterion sessions per block in which the score on type $\mathrm{N}$ trials was $80 \%$ correct or better. The type $N$ data come from 120 trials per rat (40 in each of three sessions), whereas the data on all the other variable types are from 60 trials per rat ( 20 per session).

problems. We wanted to retain the background differences in the main experiment, in order to clearly mark the change from one problem to the next, so we eliminated the highest gray level, $6.8 \mathrm{~cd} / \mathrm{m}^{2}$. From then on, the brightest gray (which formed the background in light background problems) was $5.0 \mathrm{~cd} / \mathrm{m}^{2}$.

Main experiment Phase 1: Postcriterion testing. In Phase 1 , two problems were trained to criterion, followed by two blocks of postcriterion tests. The groups learned at similar rates; on Problem 1, the FX group took, on average, 416 trials to criterion and the SH group 418, whereas on Problem 2, the groups took 576 and 569 trials, respectively, $(t \mathrm{~s}<1)$. The slower learning of these problems, as compared with those in pretraining, reflects the fact that the scenes were harder to discriminate and the criterion more stringent. Problem 2 was learned even more slowly, probably because it followed extensive postcriterion training on Problem 1.

In the first block of postcriterion tests, we compared performance within sessions on variable types $N, O P 1$, and O1P1. Data were taken from three sessions, in each of which the rat scored at least $80 \%$ correct on the baseline type $\mathrm{N}$ trials. Nearly all the rats achieved this within the first three postcriterion sessions, only a few requiring four, five, or six sessions, with no difference between the lesion groups. A second block of test sessions followed, in which types $\mathrm{N}, \mathrm{O} 2$, and $\mathrm{P} 2$ were compared; once again, nearly all the rats scored $80 \%$ correct on type $\mathrm{N}$ in each of the first three sessions, and only a few required as many as six test sessions. Thus, the lesion groups were being compared at equivalent levels of practice.

Figure 6 shows the mean performance with each of the variable types in both test blocks of Problem 1 .

Because a session was only included in the analysis if at least $80 \%$ correct choices were made on trials with variable type $\mathrm{N}$, both groups necessarily scored above $80 \%$ in this condition, in both test blocks. However, they differed in their pattern of responding with the two other variable types, OP1 and O1P1, in Block 1. The left panel of Figure 6 shows that the SH group chose less accurately with variable type OP1 than with type $\mathrm{O} 1 \mathrm{P} 1$, whereas the FX group performed similarly with both types.

A $2 \times 3$ mixed design ANOVA (groups $\times$ variable type) on the data from Block 1 yielded a significant variable type effect $[F(2,24)=8.81, p<.01]$, showing that performance with the three types was different overall, and also a group $\times$ variable type interaction $[F(2,24)=3.45$, $p<.05]$. Tests of simple effects showed that the groups did not differ in their accuracy with the baseline type $\mathrm{N}$ $[F(1,12)=1.27]$ or on type OlP1 $(F<1)$. However, the $\mathrm{SH}$ group performed marginally worse than the FX group on type OP1 $[F(1,12)=4.12, p=.06]$. Furthermore, the SH group performed much worse on OP1 than on O1P1 $[F(1,8)=12.89, p<.01]$, whereas the FX group did not $(F<1)$.

These results confirm that, as we speculated previously, normal rats see variables that share an object-position combination with the constant (OP1) as more similar to it, and therefore harder to discriminate, than the comparison O1P1 type. But the FX group did not differentiate between $\mathrm{OP} 1$ and $\mathrm{O} 1 \mathrm{P} 1$ variables, suggesting that they were less ready than the controls to encode objectposition combinations.

In the second block of tests (right panel of Figure 6), we compared variable types $\mathrm{P} 2$ (same positions, different objects) and $\mathrm{O} 2$ (same objects, different positions). Clearly, both groups saw type $\mathrm{P} 2$ variables as more similar to the constant than they did type $\mathrm{O} 2$, and the groups did not differ in this respect. A groups $\times$ variable type ANOVA showed only a significant effect of variable type $[F(2,24)=21.5, p<.001]$. Neither the group effect nor the interaction was significant $(F \mathrm{~s}<1)$. Performance was significantly worse, on the average, with $\mathrm{P} 2$ than with $\mathrm{O} 2$ variables $[F(1,12)=17.77, p<.01]$, and the difference did not interact with groups $(F<1)$. Moreover, the groups did not differ on any variable type $\left(F_{\mathrm{S}}<1\right)$. So, with the range of stimuli that we employed, the position differences were more salient than the object ones, and that was equally true of both groups.

The same comparisons of postcriterion performance between $\mathrm{OP} 1$ and $\mathrm{O} 1 \mathrm{P} 1$ and between $\mathrm{P} 2$ and $\mathrm{O} 2$ were replicated with a second problem; Figure 7 shows the results.

The left panel of Figure 7 shows that, unlike on Problem 1, neither group now differentiated between types OP1 and O1P1. There was a variable type effect $[F(2,24)=$ $14.03, p<.01]$, but there were no group or interaction effects $(F \mathrm{~s}<1)$. The variable effect arose entirely because type $\mathrm{N}$ gave higher scores than did the other two types; the difference between OP1 and OIPI was not significant $[F(1,12)=1.89]$ and did not interact with groups $(F<1)$. So the propensity to see OP 1 variables as more similar to the constant than was type O1P1, which the controls had shown in Problem 1, had disappeared. The right panel, however, shows that both groups continued to have more 


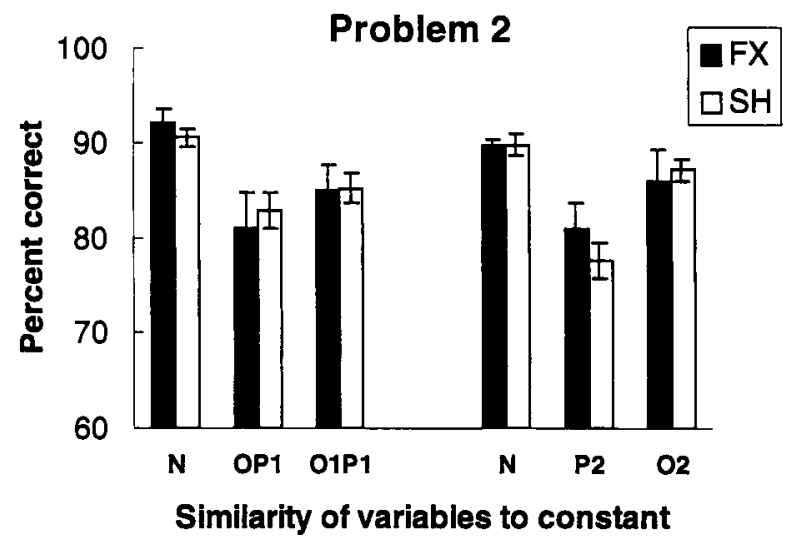

Figure 7. Experiment 1, Problem 2. Percent correct choices by the two groups (dark bars, FX; light bars, SH) on trials with different types of variable scene, in Block 1 of testing (left) and in Block 2 (right). Data are taken from three postcriterion sessions per block in which the score on type $\mathrm{N}$ trials was $80 \%$ correct or better. The type $N$ data come from 120 trials per rat (40 in each of three sessions), whereas the data on all the other variable types are from 60 trials per rat ( 20 per session).

difficulty discriminating type $\mathrm{P} 2$ from the constant than they did type $\mathrm{O} 2$. Both the overall variable type effect $[F(2,24)=19.92]$ and the difference between $\mathrm{P} 2$ and $\mathrm{O} 2$ $[F(1,12)=11.32]$ remained significant $(p s<.01)$, and neither showed any interaction with group $(F \mathrm{~s}<1)$.

Comparison of the right panels of Figures 6 and 7 shows that, for the SH controls, the difference in salience between the object and position cues was stable across the two problems; therefore, the loss of their differentiation between OP1 and O1P1 cannot be explained by a change in their sensitivity to the basic object and position cues. It must imply that their preference, in Problem 1, for encoding object-position compounds was transient. This is not very surprising, because the task did not require them to treat OP1 variables any differently from O1P1, and, by the time of the postcriterion tests, they had had long practice at discriminating the current constant scene from many types of variable scenes.

Main experiment Phase 2: Precriterion testing. In Problems 3 and 4, we asked whether the difference between variable types OP1 and O1P1, seen in the SH group in Problem 1 but not in Problem 2, would reappear if these variable types were introduced at an earlier stage of training a new problem, before the rats were highly familiar with the constant scene. As the FX rats had not shown this effect at all in either previous problem, we did not expect them to show it now.

After one preliminary session in which all the variables were of type $\mathrm{N}$, sessions comprised a mixture of 40 type N, 20 type OP1, and 20 type O1P1. The number of sessions (excluding the preliminary one) up to and including the criterion session in which the rat first scored $80 \%$ on type $\mathrm{N}$ varied from 1 to 11 but rarely exceeded 4 . The distribution was not normal. The median number of sessions for Problem 3 was 3 by Group FX and 2 by
Group SH. For Problem 4, the medians were 6 by Group FX and 3 by Group SH. The groups learned Problem 3 at similar rates (Mann-Whitney $U=21.5, n s=5$ and 9), whereas Group FX learned Problem 4 marginally slower than Group SH $(U=7.5, p<.1)$.

Because of the variable numbers of sessions to criterion both between and within groups, we analyzed the precriterion performance by splitting it into the first and second halves. From each rat, we included his sessions up to and including the criterion session but omitted any early sessions in which correct responding to type $\mathrm{N}$ was $60 \%$ or less, because this near-chance performance would merely add noise to the data. Such sessions were rarea few occurred in 3 of the FX rats and 2 of the SH rats. In Problem 3, the median number of sessions included in analysis was two, for both FX and SH groups. In Problem 4, the median numbers were five for Group FX and two for Group SH. These series of sessions were split into first and second halves. For example, if only one session of 80 trials was analyzed, the first and second halves each comprised 40 trials ( $20 \mathrm{~N}, 10 \mathrm{OP} 1,10 \mathrm{O} 1 \mathrm{P} 1)$. If there were three sessions ( 240 trials), the first and second halves each comprised one and a half sessions, or 120 trials.

Figure 8 shows the two groups' performance with variable types N, OP1, and O1P1, during the first and second halves of acquisition of Problems 3 and 4 . The main question was whether Group SH would discriminate type OP1 worse than they did type O1P1, as they had in Problem 1. Differences among variable types were tested by orthogonal planned comparisons, $\mathrm{N}$ versus the average of OP1 and O1P1 (to confirm that type $\mathrm{N}$ was easier to discriminate than the others) and OP1 versus O1P1 (the main comparison of interest).

In Problem 3, as Figure 8 suggests, there were no interesting differences either within or between groups. Trivially, both groups discriminated better during the second half than during the first half of learning. The two halves were analyzed separately in mixed design ANOVAs (group $X$ variable type). During the first half, there were no differences either between groups or among variable types $(F \mathrm{~s}<1)$. During the second half, the variable types differed $[F(2,24)=8.17, p<.01]$, but this merely reflected better discrimination with type $\mathrm{N}$ than with $\mathrm{OP} 1$ or $\mathrm{O} 1 \mathrm{P} 1$ $[F(1,12)=17.46, p<.01]$. There were no effects or interactions involving the groups $(F \mathrm{~s}<1)$.

During the first half of learning of Problem 4, Group SH performed better than Group $\mathrm{FX}[F(1,12)=11.45$, $p<.01]$, which presumably reflects their faster learning overall, noted above. Both groups scored higher with type $\mathrm{N}$ than with OP1 or O1P1 $[F(1,12)=7.69, p<$ $.05]$, and there were no differences between the latter types, although these comparisons are not really valid, given the groups' different levels of performance. During the second half, the groups performed similarly overall $[F(1,12)=1.40]$, and both did better with $\mathrm{N}$ than with OP1 or O1P1 $[F(1,12)=18.57, p<.01]$, an effect that did not interact with groups. However, as can be seen from Figure 8, the pattern of responding to OP1 and 


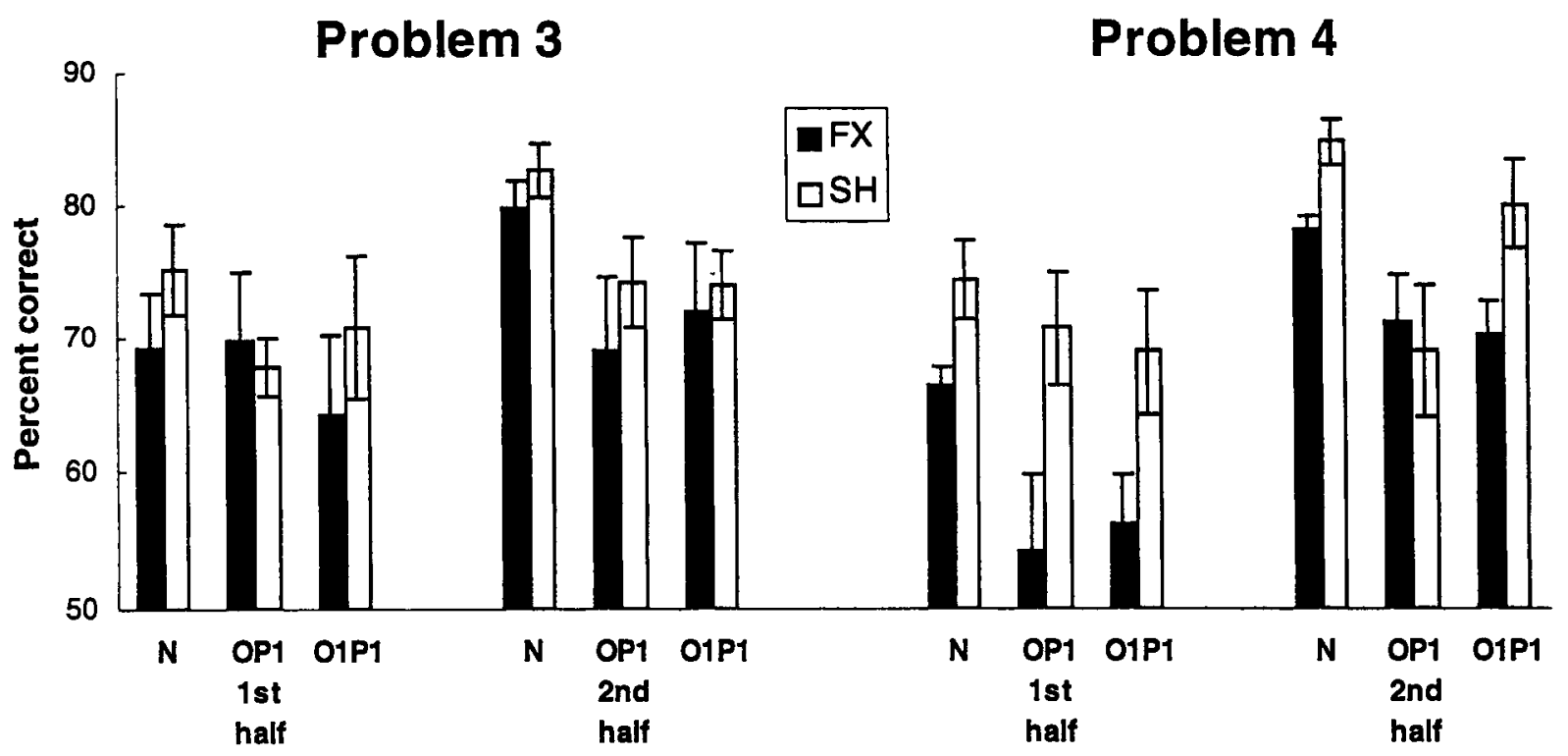

Figure 8. Experiment 1, Problems 3 and 4. Performance by the two groups with variable types N, OP1, and O1P1. All three types were presented throughout acquisition, and data are taken from sessions up to and including that in which the rat first scored $80 \%$ correct on type $\mathbf{N}$ trials. Scores are shown separately from the first and second halves of each rat's series of sessions; the total numbers of sessions analyzed varied from one to eight. See the text for details of how sessions were selected.

O1P1 was like that seen in Problem 1; Group SH performed more poorly with OP1 variables than with O1P1, whereas the FX group performed similarly with both. The planned comparisons confirmed that the difference between $\mathrm{OP} 1$ and $\mathrm{O} 1 \mathrm{P} 1$ interacted marginally with groups $[F(1,12)=3.17, p=.1]$; in the FX group, the difference was not significant $(F<1)$, but in the $\mathrm{SH}$ group it was $[F(1,8)=5.44, p<.05]$.

\section{Discussion}

Both fornix-transected and sham-operated rats readily learned the constant-negative task, although the rats in the FX group were slightly slower to acquire more difficult problems. However, for our purpose of analyzing the groups' response to different types of variable - that is, their perception of similarity between variable and constant scenes-we were generally able to compare the two groups at similar levels of performance; thus, any group differences were not confounded with different levels of expertise.

From the P2 and $\mathrm{O} 2$ conditions of Problems 1 and 2, we showed that the groups did not differ in their sensitivity either to the more salient position differences or to the less salient object differences. This supports the conclusion of E. A. Gaffan and Eacott (1997), based on a different paradigm, that fornix transection does not impair rats' discrimination of the single elemental cues, object or position, within our scenes.

The OP1 and O1P1 conditions provided evidence that object-position compound cues were more salient to the $\mathrm{SH}$ rats than to the FX rats. In Problem 1 during postcriterion testing and in Problem 4 during precriterion testing, the $\mathrm{SH}$ rats made significantly more errors in trials with
OP1 variables that shared an object-position compound with the constant scene than in trials with O1P1 variables that were equally similar to the constant in terms of the object and position elements but did not share an object-position compound. The FX rats never showed any such differentiation at any stage of the experiment, suggesting that their behavior was influenced by the shared elements only, not by the presence of a shared compound.

The effect seen in the SH rats was, of course, not very strong, and there is no obvious reason why it should appear in Problem 4 but not in Problem 3. However, the variability of the effect is not surprising when one considers that the task allowed the rats to exploit any difference between constant and variable; the rats could, in theory, learn to ignore object-position compound cues when discriminating type OP1 variables from the constant. In Experiment 2, therefore, we used a different task in which the scenes to be discriminated were designed to differ in only one way, so that the rats would have a motive to attend to that cue.

\section{EXPERIMENT 2}

In this experiment, the rats learned simple discriminations between two scenes, one rewarded and one nonrewarded. All scenes comprised two objects. Each rat learned four discriminations. In one, OPd, both the objects and the positions differed between the scenes. In the other three, the two scenes differed in one specific way. In type Od, the objects were different but the positions the same; in type $\mathrm{Pd}$, the positions were different but the objects the same; and in type OPx, the same objects and positions occurred in both scenes but were ex- 

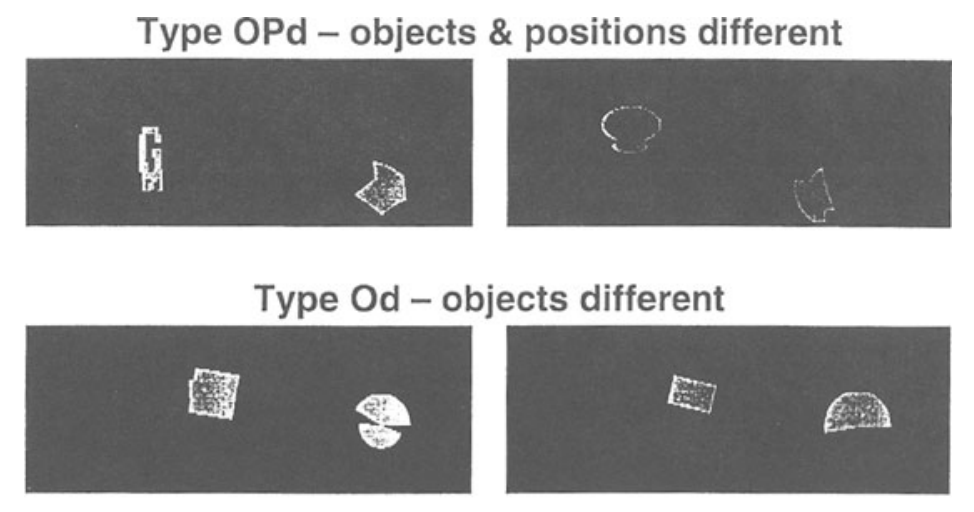

Type Pd - positions different

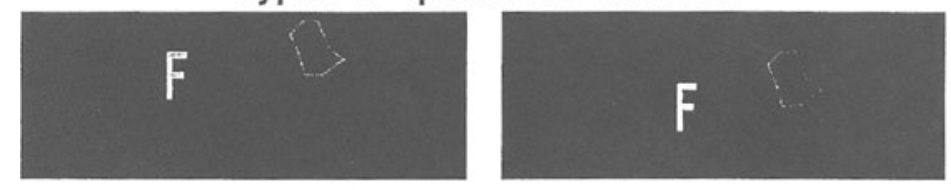

Type OPx - objects \& positions exchanged
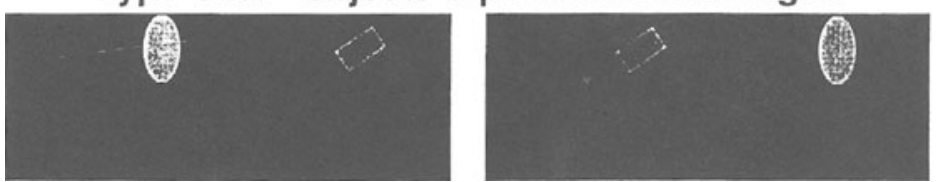

Figure 9. Examples of scenes used in Experiment 2. These four pairs of scenes- of type OPd, Od, Pd, and OPx - were used for the four discriminations learned by $1 \mathrm{FX}$ and $1 \mathrm{SH}$ rat. For details, see the text.

changed or recombined. Examples of all four types of scene pair are shown in Figure 9.

On the basis of Experiment 1, we expected that the FX and $\mathrm{SH}$ groups would perform similarly on types $\mathrm{Pd}$ and Od, but differ on type OPx.

\section{Method}

Subjects and Apparatus. All the rats who had taken part in Experiment 1 proceeded straight to Experiment 2, except for $1 \mathrm{SH}$ rat who became ill, so the group sizes were $5(\mathrm{FX})$ and $8(\mathrm{SH})$. The apparatus was the same as that for Experiment 1.

Stimuli. The stimuli were 2-object scenes, the 2 objects being on left and right screens. As previously described, each rat learned four discriminations between pairs of scenes of different types, called OPd (objects and positions different), Od (objects different), $\mathrm{Pd}$ (positions different), and OPx (objects and positions exchanged). All scenes had dark-gray backgrounds and lighter gray foreground objects, drawn from the same pool of 240 objects as that used in Experiment 1 .

Five different sets of four pairs of scenes were compiled. Each set of four pairs was assigned to 1 rat in Group FX and to either 1 or 2 rats in Group SH. One of the sets is illustrated in Figure 9. Within each set, all the objects and positions were different from each other - that is, no object or position was repeated across the four problems that were learned by 1 rat. Within a given scene, the two objects were of different types (e.g., ASCII character and polygon) and differed unsystematically in size and brightness. The intention was that, in type $\mathrm{OPx}$, the exchanged objects should be dissimilar, making it obvious that the two scenes were different from one another. Note also that the object differences in type Od and the position differences in type Pd were not very extreme. In Od, both objects at a given position were of the same type (e.g., rectangles), and, in $\mathrm{Pd}$, the positions of the matching objects were close together. The thinking behind this was that it should be beneficial to attend to both components of a scene rather than one. If the rats had developed a consistent tendency to attend only to one part of each scene, that would have obscured the distinction between type OPx and the others. However, in type OPd, which was used for baseline training and was not part of the main series of conditions to be compared (see below), no restrictions were placed on the choice of objects.

Procedure. The rats had up to now experienced only the constantnegative procedure. They were, therefore, given one practice discrimination in which the stimuli were two "easy" scenes of the kind that had been used in early pretraining with the constant-negative task, one scene rewarded and one nonrewarded, using the procedure described below. After learning this, they started the first problem of the main experiment.

The first problem was type OPd for all rats. This was designed to be easier than the other three types and was given first so that the rats would be practiced by the time they came to the main experimental conditions. It also provided a baseline index of individual learning rate, which is quite variable in this task.

Thirty trials were given in the first session and the number was progressively raised to 50 per session. The procedure was similar to that shown in Figure 4, except that correction of errors was allowed and, of course, the same two scenes were presented on every trial. Any arm could be the start arm. On the first trial of a session, the 

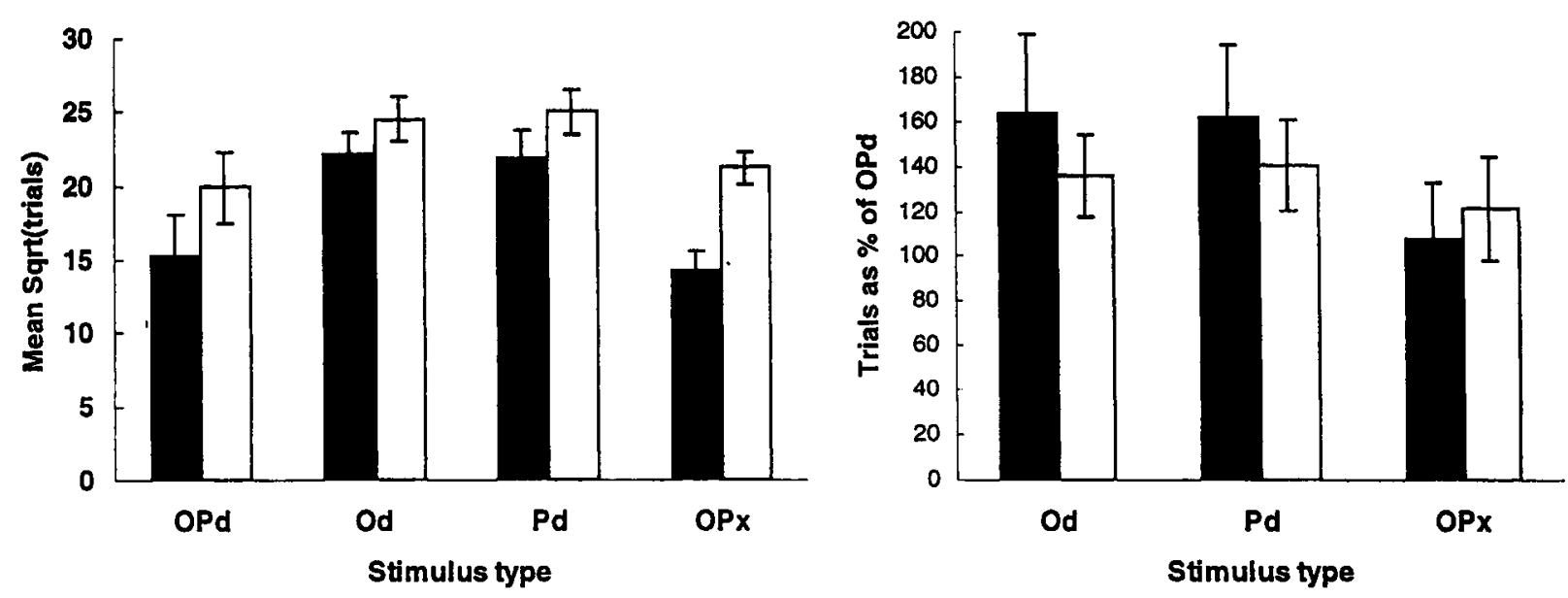

Figure 10. Experiment 2. Left panel, mean square root of trials to criterion in the four problem types, by Groups FX and SH. Right panel, trials to criterion on types Od, Pd, and OPx (square root transformed) as a percentage of the individual rat's transformed trials to criterion on type OPd.

start arm was chosen randomly and indicated by display of the horizontal stripe signal. When the rat entered the start arm, the two scenes to be discriminated were presented in the other two arms, randomly to left and right. On the first trial of a problem, it was not predefined which scene was to be rewarded; whichever scene the rat first chose was not rewarded and was defined as the nonrewarded stimulus $(\mathrm{S}-$ ) thereafter, whereas the other scene became $\mathrm{S}+$; that is, the rats were trained against initial preference.

If the rat chose the $\mathrm{S}-$ scene, both scenes were immediately turned off and a 6-sec intertrial interval commenced. Error correction was allowed; following an error, exactly the same sequence was repeated - that is, the horizontal stripe signal appeared in the same start arm as before - then $\mathrm{S}+$ and $\mathrm{S}$ - were re-presented in the same arms as before, up to three more times. If the rat made four successive errors without self-correcting, a new trial began - that is, the start arm was changed to be the arm most recently chosen-and the left-right positions of $\mathrm{S}+$ and $\mathrm{S}-$ were redetermined randomly.

If the rat chose $S+$, two food pellets were delivered while $S+$ remained on screen. The arm just chosen became the start arm for the next trial, which commenced immediately after the second pellet had been collected. Only the first occurrence of a trial was counted for the purpose of recording correct or wrong choices; that is, error corrections were ignored.

The OPd problem continued across sessions until the rat attained the criterion of $80 \%$ correct in two successive sessions. There then followed three problems - $\mathrm{Od}, \mathrm{Pd}$, and $\mathrm{OPx}-$ given in different orders to different rats. Complete counterbalancing was not possible given the group sizes of 5 and 8 , but every order used with a FX rat was given to 1 or $2 \mathrm{SH}$ rats. Each of the three problems continued, with 50 trials per session, either until criterion was attained or until 16 sessions ( 800 trials) had been completed.

\section{Results}

We counted numbers of trials to criterion, excluding the two sessions in which criterion was attained. (Total errors were also recorded but were closely correlated with trials and did not show any different effects.) The number of trials taken to learn the first (OPd) problem varied widely, ranging from 60 to 540 in Group FX and from 140 to 840 in Group SH. The distributions were skewed, so the scores were subjected to square root trans- formation before analysis. The group mean square root transformed numbers of trials for the OPd problem are shown at the extreme left of Figure 10. Group FX learned this first problem more rapidly than did Group SH but not significantly so $[t(11)=1.23]$.

We next examined whether there was any practice effect across the three problems that the rats subsequently learned. Any problems in which criterion was not reached within 16 sessions was assigned a nominal score of 800 trials. The transformed trials to criterion for each problem in order of presentation, ignoring what type it was, were analyzed with a group $\times$ order ANOVA. The FX group learned the three problems faster, on the average, than did the SH group $[F(1,11)=7.29, p<.05]$, but there was no effect of order, nor did it interact with group $\left(F_{\mathrm{s}}<1\right)$. We, therefore, did not take into account order of learning when analyzing the $\mathrm{Od}, \mathrm{Pd}$, and $\mathrm{OPx}$ conditions.

The left panel of Figure 10 also shows the mean transformed trials to criterion from those three conditions. From inspection, there is a clear group difference in rate of learning the OPx problem but not any other type. The difference takes the form that the FX group learned faster than the $\mathrm{SH}$ group. This will be discussed later, but it must first be qualified in light of the fact that the FX group generally learned faster throughout, as reported above. To adjust for this, and also for the large individual differences in learning rate that tended to be consistent across problems, we expressed the (square root transformed) trials to criterion in each of the Od, Pd, and OPx problems as a percentage of the same rat's transformed trials to criterion in the baseline OPd problem.

The mean percentage values of the two groups are shown in the right panel of Figure 10. The percentage conversion tends to equalize the groups' average score; however, it is still clear that the problem types differed in relative difficulty. Whereas the $\mathrm{SH}$ group learned all three types of problem at a similar rate $(120 \%-140 \%$ of 
the OPd baseline), the FX group found the Od and Pd types relatively hard and the OPx type relatively easy.

A group $\times$ problem type ANOVA was applied to the percentage scores. The group main effect was nonsignificant $(F<1$; nor did the groups differ on any individual problem type; $F_{\mathrm{s}}<1$ ), but there was a significant effect of problem type $[F(2,22)=6.96, p<.05]$. The problem type effect was split into orthogonal contrasts-OPx versus the average of the two singleelement types $\mathrm{Od}$ and $\mathrm{Pd}$ and $\mathrm{Od}$ versus $\mathrm{Pd}$. The first of these contrasts was significant overall $[F(1,11)=16.05$, $p<.01]$ and interacted with groups $[F(1,11)=4.60$, $p=.05]$, indicating that the difference between OPx and the other types was greater in the FX group than in the SH controls. The second contrast between Od and Pd was not significant and did not differ between groups $(F \mathrm{~s}<1)$. Analyses of simple effects within the groups confirmed that Group FX learned the OPx problem faster than $\operatorname{Od}$ and $\operatorname{Pd}[F(1,4)=22.09, p<.01]$, whereas, in Group SH, there was not any difference between OPx and the other types $[F(1,7)=1.91]$.

\section{Discussion}

In this experiment, the simple object and position cues were more similar in salience than they had been in Experiment 1 , because we made the position cue harder; the positions of corresponding objects in condition $\mathrm{Pd}$ were much closer than in condition $\mathrm{O} 2$ of Experiment 1 (cf. Figures 2 and 9). As a result, the rates of learning with the single cues in problem types $\mathrm{Od}$ and Pd were very similar in Experiment 2, whereas performance had differed between $\mathrm{P} 2$ and $\mathrm{O} 2$ of Experiment 1 . Nonetheless, we found once again that the FX and SH groups did not differ in response to either of the simple cues, object or position, even when the position cue was made less salient.

Despite their similarity in learning about the object and position cues per se, the groups differed in condition $\mathrm{OPx}$, in which objects and positions were interchanged between the two scenes. Group FX found this condition relatively easy, as compared with $\mathrm{Od}$ and $\mathrm{Pd}$, whereas Group SH learned all three problem types at similar rates. This result confirmed our expectation that the $\mathrm{OPx}$ condition should differentiate the groups, but the direction of the difference was at first sight surprising. A simple extrapolation from conditions OP1 and O1P1 of Experiment 1 might suggest that, if the rats in the FX group are poor at encoding object-position compounds and attend primarily to the elements, they should find $\mathrm{OPx}$ pairs relatively hard to discriminate, because these pairs of scenes differ in their compounds but not their elements; but we found the opposite result.

Is it possible to reconcile the results of the two experiments? Both the OP 1 condition of Experiment 1 and the OPx condition of Experiment 2 manipulated objectposition compounds, but in a different way. In OP1, the pairs of scenes comprised one identical compound and one completely different one; in OPx, all the elements of the two scenes were similar but they were differently compounded. So OPx is a configural discrimination in the sense of Rudy and Sutherland (1995), but OP1 is not. In several studies, fornix transection has produced either mild or no impairment of various configural tasks (McDonald et al., 1997; Sziklas, Lebel, \& Petrides, 1998) but has not enhanced them. However, Bussey, Warburton, Aggleton, and Muir (1998) recently reported that fornix transection facilitated acquisition of a configural problem (transverse patterning). Their task had some similarity to our OPx procedure, not only in using computergenerated visual stimuli, but in asking the rats to choose between two simultaneously presented displays. Why might fornix transection, surprisingly, aid rats in learning some simultaneous configural discriminations?

One hypothesis, for our task, is that awareness of objectposition compounds makes the two scenes in type OPx appear similar, as well as providing the basis for telling them apart. A glance at the example in Figure 9 will confirm this impression of similarity, to human eyes. So, if the rats in Group SH are more aware of object-position compounds (as we inferred from Experiment 1), that might cause them to find the OPx discrimination difficult. To perceive similarity between the scenes, one must attend to both objects; that prevents one from acquiring a strategy that could make the task artificially easynamely, learning to attend to only one side of the scenes (effectively turning type OPx into an easy, nonconfigural object discrimination). It is possible, though we have no direct evidence, that Group FX acquired such a strategy more readily than did Group SH. The variability of learning rate observed in this experiment is consistent with the idea that these problems can be learned in more than one way.

In short, although we conceived both the OP1 and the OPx conditions as tests of the ability to encode objectposition compounds, there are other important differences between the tasks-in particular, that OPx is a configural discrimination that lends itself to solution in nonconfigural terms. In both conditions, Group FX actually discriminated better than the controls, and this could be explained by the normal rats' perceiving similarities that the lesioned rats do not, but for different reasons in the two tasks.

\section{GENERAL DISCUSSION}

First, these experiments show that rats can learn and reliably perform discriminations among purely visual abstract scenes, analogous to those used in experiments with monkeys, and that quite subtle aspects of the scenes can be manipulated in order to study rats' visuospatial encoding. We believe that the design of our apparatus, which presents wide-angle displays some distance away from the choice point, is important in revealing these abilities (E. A. Gaffan \& Woolmore, 1996).

We confirmed previous findings (E. A. Gaffan \& Eacott, 1997) that fornix transection has no perceptual effects on rats' ability to discriminate the simple cues of ob- 
ject quality or spatial location within a visual scene. The absence of a spatial cue deficit contrasts with other findings mentioned in the introduction (see, e.g., DeCoteau \& Kesner, 1998; Parkinson et al., 1988). This suggests a difference between scenes viewed from a distance (as in our apparatus) and scenes that the animal can approach and interact with. This point is discussed more fully below.

However, both our experiments suggested that fornix transection affects rats' sensitivity to object-place compound cues. Experiment 1 clearly implied that the rats in the FX group were less sensitive to such cues than those in the SH group, because Group SH sometimes treated pairs of scenes that shared a specific object-place combination (type OP1) as being more similar than were comparable pairs that did not (O1P1), whereas the FX group never treated the two types differently. In Experiment 2, Group FX seemed better able than Group SH to discriminate scenes that consisted of spatial rearrangements of the same objects. An interpretation of this paradoxical result that is consistent with the results of Experiment 1 is that sensitivity to object-place compounds makes such pairs of scenes appear similar, creating a difficulty for the controls that can more easily be ignored by the lesioned group.

We can cast more light on these phenomena by using scenes that contain more than two objects, so a wider variety of complete and partial rearrangements and substitutions of objects is possible. Rats are able to learn the constant-negative task using more complex scenes with three or four objects, so it will be possible to test the generality of the present results.

What is the relationship between the effects seen here and the lesion effects on object-place encoding and memory in other kinds of task, as described in the introduction? This is not a simple question, because there are substantial differences between the procedures. One, mentioned previously, is that all the other tasks, whether they used vertical visual displays (D. Gaffan's studies with monkeys and all the experiments with humans) or three-dimensional objects distributed in a horizontal plane (Ennaceur et al., 1997; Long \& Kesner, 1995; Parkinson et al., 1988; Save et al., 1992), had the display area fixed relative to surrounding cues, so that the spatial cues being manipulated were allocentrically constant. That was not true in our apparatus, where a given scene could appear in any of the three maze arms. Another potentially important difference is that, in most of the other studies, including all those with rats and monkeys, the subject's discriminative response consisted of directed movement, usually toward a specific object within the scene. Our paradigms are different, because the rat makes its choice between scenes from some distance away, on the basis of an egocentric view of each whole scene, and is not required to contact any part of the scene to obtain reward.

Many researchers have argued that allocentric spatial coding and/or a record of the subject's own movements through space (path integration) are central components in the hippocampal influence on spatial learning (McNaughton et al., 1996; Morris, Garrud, Rawlins, \& O'Keefe, 1982; O'Keefe \& Nadel, 1978; Whishaw, McKenna, \& Maaswinkel, 1997). D. Gaffan (in press) has recently proposed that idiothetic cues generated by self-movementwhether of the whole body, limbs, or eyes - could, in theory, participate in object-in-place memory, as he has defined it in monkeys and people. The method of studying object-in-place encoding within visual scenes that we describe in this paper is distinctive because, unlike all the others, it does not obviously engage either allocentric place cues or idiothetic cues. Of course, such cues are present, but they are not relevant to the rats' performance in the tasks. If further research confirms our suggestion that this form of object-in-place encoding is dependent on an intact hippocampal system, it would provide support for the idea that the hippocampal representation of the world need not be intrinsically allocentric or linked to idiothetic cues.

\section{REFERENCES}

Aggleton, J. P. (1996). The ability of different strains of rats to acquire a visual nonmatching-to-sample task. Psychobiology, 24, 44-48.

Aggleton, J. P., \& SAHGal, A. (1993). The contribution of the anterior thalamic nuclei to anterograde amnesia. Neuropsychologia, 31, $1001-1019$.

Angeli, S. J., Murray, E. A., \& Mishkin, M. (1993). Hippocampectomized monkeys can remember one place but not two. Neuropsychologia, 31, 1021-1030.

Bussey, T. J., Warburton, E. C., Aggleton, J. P., \& Muir, J. L. (1998). Fornix lesions can facilitate acquisition of the transverse patterning task: A challenge for "configural" theories of hippocampal function. Journal of Neuroscience, 18, 1622-1631.

Cassaday, H. J., \& GafFan, E. A. (1996). A novel test of visual learning in the rat: Effects of 8-OH-DPAT and WAY-100579. Journal of Psychopharmacology, 10, 195-205.

DeCoteau, W., \& Kesner, R. P. (1998). Effects of hippocampal and parietal cortex lesions on the processing of multiple object scenes. Behavioral Neuroscience, 112, 68-82.

EnNaCEUR, A., NeAve, N., \& AgGLeton, J. P. (1997). Spontaneous object recognition and object location memory in rats: The effects of lesions in the cingulate cortices, the medial prefrontal cortex, the cingulum bundle and the fornix. Experimental Brain Research, 113, 509-519.

Gaffan, D. (1992a). Amnesia for complex naturalistic scenes and for objects following fornix transection in the rhesus monkey. European Journal of Neuroscience, 4, 381-388.

GafFAN, D. (1992b). The role of the hippocampus-fornix-mammillary system in episodic memory. In L. R. Squire \& N. Butters (Eds.), Neuropsychology of memory (2nd ed., pp. 336-346). New York: Guilford.

GafFan, D. (1994). Scene-specific memory for objects: A model of episodic memory impairment in monkeys with fornix transection. Journal of Cognitive Neuroscience, 6, 305-320.

GAFFAN, D. (in press). Idiothetic input into object-place configuration as the contribution to memory of the monkey and human hippocampus: A review. Experimental Brain Research.

GafFan, D., \& Gaffan, E. A. (1991). Amnesia in man following transection of the fornix: A review. Brain, 114, 2611-2618.

GAFFAN, D., \& HaRRISON, S. (1989a). A comparison of the effects of fornix transection and sulcus principalis ablation upon spatial learning by monkeys. Behavioural Brain Research, 31, 207-220.

GafFan, D., \& Harrison, S. (1989b). Place memory and scene memory: Effects of fornix transection in the monkey. Experimental Brain Research, 74, 202-212.

Gaffan, D., \& Saunders, R. C. (1985). Running recognition of con- 
figural stimuli by fornix-transected monkeys. Quarterly Journal of Experimental Psychology, 37B, 61-71.

GafFan, E. A., \& EacotT, M. J. (1995). A computer-controlled maze environment for testing visual memory in the rat. Journal of Neuroscience Methods, 60, 23-37.

GafFan, E. A., \& EacotT, M. J. (1997). Spatial memory impairment in rats with fornix transection is not accompanied by a simple encoding deficit for directions of objects in visual space. Behavioral Neuroscience, 111, 937-954.

GafFan, E. A., \& WoOlmore, A. L. (1996). Complex visual learning by rats. Learning \& Motivation, 27, 375-399.

Hornak, J., Oxbury, S., Oxbury, J., Iversen, S., \& Gaffan, D. (1997). Hemifield-specific visual recognition memory impairments in patients with unilateral temporal lobe removals. Neuropsychologia, 35, 1311-1315.

JARRARD, L. E. (1995). What does the hippocampus really do? Behavioural Brain Research, 71, 1-10.

Kapur, N., Scholey, K., Barker, S., Mayes, A., Brice, J., \& FlemING, J. (1994). The mammillary bodies revisited: Their role in human memory functioning. In L. S. Cermak (Ed.), Neuropsychological explorations of memory and cognition: Essays in honor of Nelson Butters (pp. 159-189). New York: Plenum.

LONG, J. M., \& KeSNER, R. P. (1995). The effects of hippocampal and parietal cortical lesions on memory for an object/spatial location paired associate task. Society for Neuroscience Abstracts, 21, 1215.

Malkova, L., \& Mishkin, M. (1997). Memory for the location of objects after separate lesions of the hippocampus and parahippocampal cortex in rhesus monkeys. Society for Neuroscience Abstracts, $23,12$.

McDonald, R. J., Murphy, R. A., Guaracci, F. A. Gortler, J. R., WHITE, N. M., \& BAKER, A. G. (1997). Systematic comparison of the effects of hippocampal and fornix-fimbria lesions on acquisition of three configural discriminations. Hippocampus, 7, 371-388.

McNaughton, B. L., Barnes, C. A., Gerrard, J. L., Gothard, K., Jung, M. W., Knierim, J. J., Kudrimoti, H., Qin, Y., SKaGgS, W. E., Suster, M., \& Weaver, K. L. (1996). Deciphering the hippocampal polyglot: The hippocampus as a path integration system. Journal of Experimental Biology, 199, 173-185.

Milner, B., JohnSRUDE, I., \& CRANE, J. (1997). Right medial temporallobe contribution to object-location memory. Philosophical Transactions of the Royal Society of London: Series B, 352, 1469-1474.

MorRIS, R. G. M., GARRUD, P., RAWLINS, J. N. P., \& O'KEEFE, J. (1982). Place navigation impaired in rats with hippocampal lesions. Nature, 297, 681-683.
Nadel, L., Allen, J. J. B., Bohbot, V. D., Kalina, M., \& StepanKovA, K. (1997). Hippocampal and parahippocampal role in processing spatial configurations in humans-ERP and lesion data. Society for Neuroscience Abstracts, 23, 2108.

O'KeEFE, J., \& NADEL, L. (1978). The hippocampus as a cognitive map. Oxford: Oxford University Press.

Owen, A. M., Milner, B., Petrides, M., \& Evans, A. C. (1996). A specific role for the right parahippocampal gyrus in the retrieval of object-location: A positron emission tomography study. Journal of Cognitive Neuroscience, 8, 588-602.

PARKER, A., \& GAFFAN, D. (1997a). The effect of anterior thalamic and cingulate cortex lesions on object-in-place memory in monkeys. Neuropsychologia, 35, 1093-1102.

Parker, A., \& Gaffan, D. (1997b). Mamillary body lesions in monkeys impair object-in-place memory: Functional unity of the fornixmamillary system. Journal of Cognitive Neuroscience, 9, 512-521.

Parkinson, J. K., Murray, E. A., \& Mishkin, M. (1988). A selective mnemonic role for the hippocampus in monkeys: Memory for the location of objects. Journal of Neuroscience, 8, 4159-4167.

Pigott, S., \& Milner, B. (1993). Memory for different aspects of complex visual scenes after unilateral temporal- or frontal-lobe resection. Neuropsychologia, 31, 1-15.

Rudy, J. W., \& SUTherLand, R. J. (1995). Configural association theory and the hippocampal formation: An appraisal and reconfiguration. Hippocampus, 5, 375-389.

Save, E., Poucet, B., Foreman, N., \& Buhot, M.-C. (1992). Object exploration and reactions to spatial and nonspatial changes in hooded rats following damage to parietal cortex or hippocampal formation. Behavioral Neuroscience, 106, 447-456.

SMITH, M. L., \& MILNER, B. (1989). Right hippocampal impairment in the recall of spatial location: Encoding deficit or rapid forgetting? Neuropsychologia, 27, 71-81.

Sutherland, R. J., \& Rodriguez, A. J. (1989). The role of the fornix/fimbria and some related subcortical structures in place learning and memory. Behavioural Brain Research, 32, 265-277.

Sziklas, V., Lebel, S., \& Petrides, M. (1998). Conditional associative learning and the hippocampal system. Hippocampus, 8, 131-137.

Whishaw, I. Q., McKenna, J. E., \& MaAsWinkel, H. (1997). Hippocampal lesions and path integration. Current Opinion in Neurobiology, 7, 228-234.

(Manuscript received April 15, 1998; revision accepted for publication June 24,1998 .) 